\title{
Evaluation of a class of NLFM radar signals
}

\author{
Sebastian Alphonse* (1) and Geoffrey A. Williamson
}

\begin{abstract}
Signal design is an important component for good performance of radar systems. Here, the problem of determining a good radar signal with the objective of minimizing autocorrelation sidelobes is addressed, and the first comprehensive comparison of a range of signals proposed in the literature is conducted. The search is restricted to a set of nonlinear, frequency-modulated signals whose frequency function is monotonically nondecreasing and antisymmetric about the temporal midpoint. This set includes many signals designed for smaller sidelobes including our proposed odd polynomial frequency signal (OPFS) model and antisymmetric time exponentiated frequency modulated (ATEFM) signal model. The signal design is optimized based on autocorrelation sidelobe levels with constraints on the autocorrelation mainlobe width and leakage of energy outside the allowed bandwidth, and we compare our optimized design with the best signal found from parameterized signal model classes in the literature. The quality of the overall best such signal is assessed through comparison to performance of a large number of randomly generated signals from within the search space. From this analysis, it is found that the OPFS model proposed in this paper outperforms all other contenders for most combinations of the objective functions and is expected to be better than nearly all signals within the entire search set.
\end{abstract}

Keywords: Radar signal processing, Signal design, Matched filters, Frequency modulation, PSLR, ISLR

\section{Introduction}

Radar's primary objective is to detect targets and estimate their range and velocity. To achieve these objectives, it is desirable that the radar signals have small autocorrelation (AC) sidelobes or Doppler tolerant. Optimizing over one characteristic can yield other undesired effects such as widening the AC mainlobe. Achieving a balance between the conflicting requirements is the goal of signal modulation. Radar signals are either frequency modulated or phase modulated. In this paper, we address finding FM signals that have high-quality performance on reducing the sidelobes with the constraints on mainlobe width and energy leakage beyond the specified bandwidth.

The most popular frequency modulation (FM) signal is linear frequency modulation (LFM). Though LFM has good Doppler tolerant characteristic, it has relatively high sidelobes $(-13.4 \mathrm{~dB})$ which may not be suited for many other radar applications. To achive smaller

*Correspondence: salphons@hawk.iit.edu

Department of Electrical and Computer Engineering, Illinois Institute of

Technology, Chicago, IL, 60616, USA
AC sidelobes and also to maintain constant envelope, researchers turned to stationary phase principle (SPP) in which the frequency function is varied nonlinearly to shape the PSD to one that corresponds to smaller AC sidelobes.

Using this principle, Cook [1] derived frequency functions whose PSDs have the shape of an $n$th power of cosine. Cook also noted that deriving the frequency function from the PSDs can be done only for relatively simple PSDs, and in his paper [1], Cook derived frequency functions only for $n=1,2,3$, and 4 . The best peak sidelobe level he reported was of $-47 \mathrm{~dB}$ for the choice of $n=4$. The limitation of SPP to use only simple PSDs led researchers to come up with ad hoc frequency functions that have desirable PSDs resulting in smaller AC sidelobes. In 1979, Price proposed a nonlinear frequency function that achieves small sidelobes [2], ([3], Section 5.2), ([4], Section 8.2.2). After Price's nonlinear frequency modulation (NLFM), many researchers proposed ad hoc frequency functions with the aim of achieving smaller AC sidelobes [5-9]. These NLFM signals are from the signal set $\Omega$ : these signals have monotonically increasing and 
midpoint antisymmetric frequency functions. These frequency functions have a continuously varying first derivative, except for specific parameter settings in which case they result in LFM.

There is another class of NLFM signals that also belongs to the signal set $\Omega$ but whose frequency functions have three segments [10-13]. In the middle segment, the frequency is a linear function of time having constant first derivative. The first and the third segments have derivatives diverging from this particular constant value. Cook [10] proposed such an NLFM signal whose frequency function has three piecewise linear components and achieved a peak sidelobe level below $-30 \mathrm{~dB}$. Though in this case the first and third components are linear, Cook suggested that these components can be nonlinear. Griffiths and Vinagre [11] used this piecewise linear FM signal and applied amplitude modulation to get further reduction in sidelobe levels. Other researchers suggested nonlinear functions for the first and third segments to achieve reduced sidelobe levels without performing amplitude modulation $[12,13]$.

\subsection{Objective functions}

We are interested to find the "best" NLFM signal with small sidelobes. To do this, we define a family of objective functions that penalize AC sidelobes. The AC of signal $s$ is defined as

$$
R_{s}(m)= \begin{cases}\sum_{n=0}^{M-m-1} s(n+m) s^{*}(n), & m \geq 0, \\ R_{s}^{*}(-m), & m<0 .\end{cases}
$$

The papers we have discussed so far focus on minimizing only peak sidelobe level with respect to the mainlobe level. Another important performance metric often considered in the literature is the integrated sidelobe ratio (ISLR), which is the energy in sidelobes with respect to the energy in the mainlobe [14-16]. It is reasonable to form objective functions that are convex combinations of ISLR and the peak-to-sidelobe ratio (PSLR). By choosing the convex weighting between zero to one, the radar operator can emphasize one metric over the other.

PSLR is defined as the ratio of maximum sidelobe level squared with respect to the mainlobe level squared. If we denote the $p$ th AC sidelobe peak value as $R_{p}$, then

$$
\operatorname{PSLR}(s)=\left(\max _{p}\left\{R_{p}\right\}\right)^{2} / R_{s}^{2}(0) .
$$

There are few slightly varying definitions for ISLR used in the literature. The one we have used in this paper is the one that is widely used $[15,17,18]$ in which, the ISLR is defined as the ratio of energy in the $\mathrm{AC}$ when the signals are not time aligned to the energy in $\mathrm{AC}$ when the signals are time aligned. In this case, one sample correspond to one sample delay in autocorrelation function.

$$
\operatorname{ISLR}(s)=\left(\sum_{\substack{m=-M \\ m \neq 0}}^{M} R_{s}^{2}(m)\right) / R_{s}^{2}(0) .
$$

A signal with small sidelobes will have smaller values for both PSLR and ISLR. We form a cost function as the convex combination of these two metrics. Note that PSLR is always less than one and ISLR can be greater than one depending on the signal model. This will force the optimization procedure to give more importance in minimizing ISLR. To bring both these objectives to a comparable level, we will normalize PSLR and ISLR with respect to LFM's PSLR and ISLR:

$$
\operatorname{NPSLR}(s)=\frac{\operatorname{PSLR}(s)}{\operatorname{PSLR}_{L F M}}
$$

and

$$
\operatorname{NISLR}(s)=\frac{\operatorname{ISLR}(s)}{\operatorname{ISLR}_{\mathrm{LFM}}} .
$$

PSLR $_{L F M}$ and ISLR $R_{L F M}$ are the PSLR and ISLR of the LFM signal whose frequency is a linear function of time. Hence, our cost function to minimize is

$$
Q(s ; \beta)=\beta \cdot \operatorname{NPSLR}(s)+(1-\beta) \cdot \operatorname{NISLR}(s),
$$

where $\beta \in[0,1]$ is a weight parameter.

While minimizing PSLR and ISLR, the radar designer also considers the AC mainlobe width and amount of transmitted signal energy contained in the allowed bandwidth. Reducing the sidelobe level widens the mainlobe. Though reducing the sidelobes is a higher priority, having a very wide mainlobe reduces the range accuracy of the target [19]. Also. a wide AC mainlobe of a signal reflected from a strong target will mask the reflection from a weak target. Hence, we will impose a constraint that the AC mainlobe width of the signal (MLW $(s)$ ) should not be larger than twice the mainlobe width of LFM $\left(\operatorname{MLW}(s) / M_{L W} \leq 2\right)$, and we optimize $Q(s ; \beta)$ in (6) subject to this constraint.

A second constraint is about the spectral bandwidth occupancy of the signal. As the available spectrum is limited and often needs to be shared with other applications, it is important to make sure that the designed signal utlizes the available bandwidth effectively [20,21]. A signal model that occupies a larger bandwidth can have a smaller PSLR, a smaller ISLR, and a narrower mainlobe width compared to its own version occupying a smaller bandwidth. Hence, to compare the AC characteristics fairly for different signals, we have to make sure that most of their spectral energy is confined within the specified useful 
bandwidth $B_{0}$. To quantify the signal's spectral occupancy, we will define in-band energy ratio (IBER) as

$$
\operatorname{IBER}(s)=\frac{\text { Energy of } s \text { in the spectral range }\left[-B_{0} / 2, B_{0} / 2\right]}{\text { Total energy of the signal } s} .
$$

In this paper, we will limit our search for the best signal within the set whose signals have at least $90 \%$ of the signal energy within the specified bandwidth (IBER $\geq 0.9$ ). It should be noted that the mainlobe width factor (two) and IBER (0.9) depend on the particular applications and the choices we have made in this paper fall under a reasonable range of values for these parameters.

\subsection{Related work on finding good FM signals with small sidelobes}

When the radar operator chooses a signal for his objective, it is useful to have a performance comparison of available radar signal designs. Though many papers have proposed different NLFM signal models, there has been little focus on comparing these signal models. Boukeffa et al. [22] compares different NLFM signal models, but there are some discrepancies in the results. For example, according to [22], the peak sidelobe level for Cook's [1] proposed signal whose PSD has the shape of cosine to the power four is $-84 \mathrm{~dB}$. But Cook himself reported the peak sidelobe level as $-47 \mathrm{~dB}$. It is not clear from these papers, how they obtained these values. We used $10 \log _{10}((\operatorname{PSLR}(s))$ $(\operatorname{PSLR}(s)$ from Eq. (2)), which is the common expression for computing PSLR in decibel. Wenzhen and Yan [7] compared several NLFM signal models (including Price's signal model) along with their own proposed NLFM signal. Though in their comparison, their proposed signal model achieved smaller sidelobes, we observed from our analysis that Price's signal model outperforms the signal model proposed in [7].

\subsection{Our contribution}

In this paper, we present the odd polynomial frequency signal (OPFS) and the asymmetric time exponentiated FM (ATEFM) signal. Polynomial-phase signals (PPS) are common in radar applications. As the name suggests in PPS, the polynomial represents the phase of the signal. While designing NLFM, representing the frequency function as a polynomial gives direct control over the PSD which helps to reduce AC sidelobes. An important distinction of OPFS is that even powers to set zero due to the antisymmetric nature of the frequency functions from the signal set $\Omega$. Hence we reference this signal as odd polynomial frequency signal (OPFS).

From our experiments, we observed that extending the frequency function beyond the allowed bandwidth range helps in reducing the $\mathrm{AC}$ sidelobe levels. In the proposed signal model, we incorporate a frequency scaling parameter which helps in extending the frequency function beyond the allowed bandwidth range. Care must be taken to limit this extension to focus most of the signal energy within the allowed bandwidth. Through simulation, we will demonstrate that OPFS outperforms other signals for most of the weighted combinations of objective functions that we consider. And the signal proposed by Price [2] outperforms the other signals when only minimizing PSLR.

The best signal in $\Omega$ may not be from any of the parameterized signal subsets that we studied. To assess the likelihood of a better signal within $\Omega$ from outside the parameterized subsets we have considered, we examine randomly selected signals in $\Omega$ drawn according to a uniform probability distribution. In Section 4, we discuss how we generate such signals. We created one million random signals and determined the best signal among them with respect to each choice of weights for the objective function. We observed that our 6D-OPFS and Price's signal still outperform these randomly picked signals. From this experiment, as discussed in Section 4, we estimate with 95\% confidence that 6D-OPFS and Price's signal outperforms $99.9997 \%$ of the signals in $\Omega$.

\section{Library of parameterized NLFM signals}

The general form for radar signals is $s(t)=A(t)$ $\exp (j \phi(t))$, where $A(t)$ is the time dependent amplitude of the signal. A varying envelope of the signal reduces transmitter efficiency. In this paper, we will assume that the signals have constant amplitude $(A(t)=A) . \phi(t)$ is the phase of the signal. Depending on the search range of the radar and desired range resolution, the radar signal is limited to have a pulse duration $T$, bandwidth $B$, and carrier frequency $f_{c}$. It is easier to design the signal in baseband, and hence, without loss of generality, we will set $f_{c}$ to zero.

The phase of the signal $\phi(t)$ can be written as an integral of the frequency function $f(t)$. The frequency functions of the FM signals proposed in the literature for small sidelobes are nondecreasing and antisymmetric about the temporal midpoint, and we restrict consideration to frequency functions of this form. The class of frequency functions $f(t)$ we consider thus satisfy the following conditions:

(A) $f(-T / 2)=-B / 2$.

(B) $f(t)$ is monotonically nondecreasing.

(C) $f(-t)=-f(t)$.

We label the set of (baseband) radar signals whose elements $f(t)$ satisfy the above three conditions as $\Omega$. Note that the bandwidth parameter $B$ that is used in signal design can be bigger than the useful bandwidth $B_{0}$ 


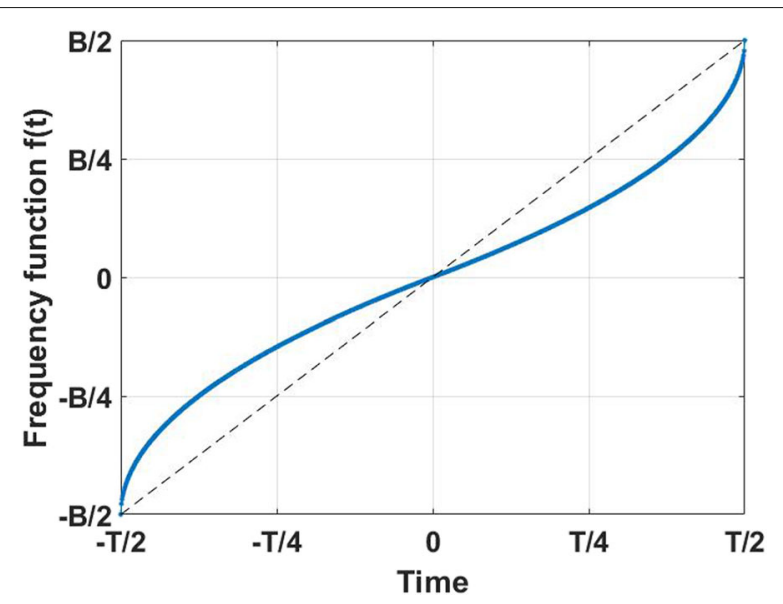

Fig. 1 Typical frequency function from the NLFM signal set $\Omega$, which is monotonically nondecreasing and has temporal midpoint antisymmetry

(defined in (7)), but care must be taken during design to make sure that the significant part of the signal energy is contained within the useful bandwidth.

In Fig. 1, we show an example of a frequency function from the signal set $\Omega$ that typifies the NLFM frequency functions proposed in the literature designed for smaller sidelobes. By studying the frequency characteristics of this typical signal, we can understand why the signals from the signal set $\Omega$ yield smaller sidelobes. The first difference which is proportional to first order derivative of this typical frequency function with respect to time is shown in

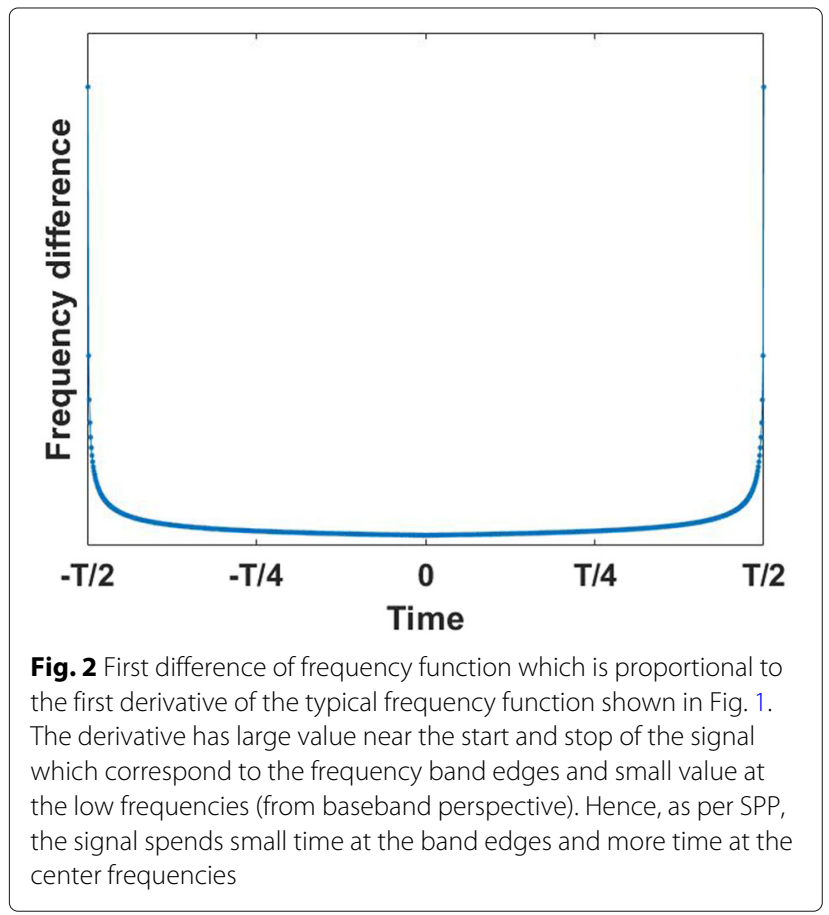

Fig. 2. We can observe that a typical frequency function has high derivative at the start and end of the signal. The spectral energy at the edges of the frequency band arises from these fast variations at the start and end of the signal. Moreover, the frequency function has a small derivative in the temporal middle of the signal duration, corresponding to the low frequencies (from a baseband perspective). This means the frequency function spends less time in the edge frequencies and more time in the low frequencies. As per the SPP, this results in smaller energy in edge frequencies and higher energy in the low frequencies as we can see in Fig. 3. As a result, we get a windowed PSD which yields smaller AC sidelobes as shown in Fig. 4. We can observe that the red (lower) horizontal line in Fig. 4 is the peak sidelobe level of this typical NLFM signal which is substantially smaller compared to the peak sidelobe level of LFM shown as the black (upper) horizontal line.

Each of the NLFM signal models proposed in the literature is characterized by its own set of parameters $\Theta$ that determine the specific nonlinear characteristics of the frequency function. In this section, we discuss the two signal models proposed by us the authors. The other signal models that we have selected from the literature for comparison are discussed in Appendix for reference. We made minor modifications to the notations to be in consistent with our notations. We have selected what we feel are the most representative among the classes of NLFM signals designed for smaller sidelobes published in the literature. We label the sets of signal models as $\Omega_{A}, \Omega_{B}, \ldots, \Omega_{H}$. However, there are other signal models that we do not

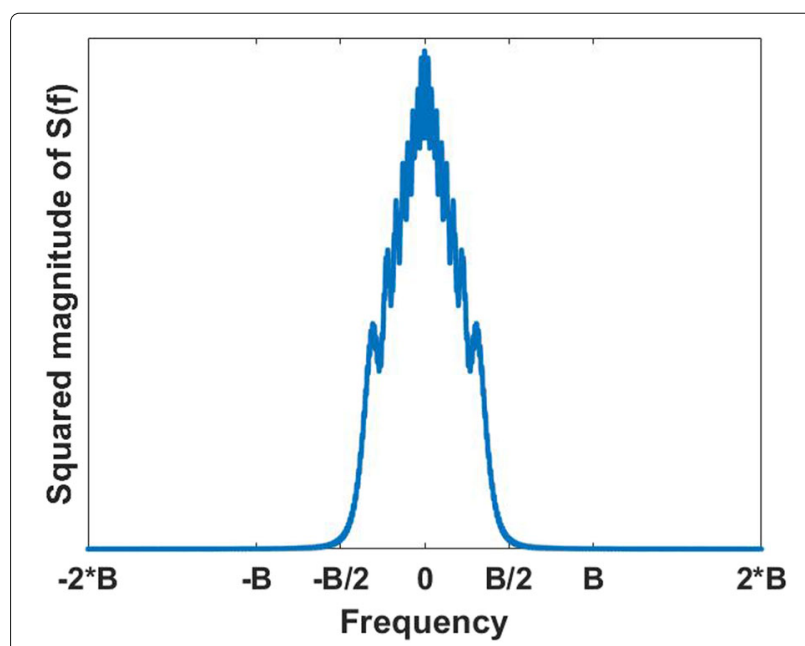

Fig. 3 Squared magnitude of fast Fourier transform which is proportional to PSD of the typical frequency function shown in Fig. 1. Edge frequencies have small PSD values and low frequencies have large PSD values. This agrees with SPP, as the edge frequencies have large derivatives and middle frequencies have large derivatives. In the end, we get a windowed PSD that should result in small AC sidelobes 


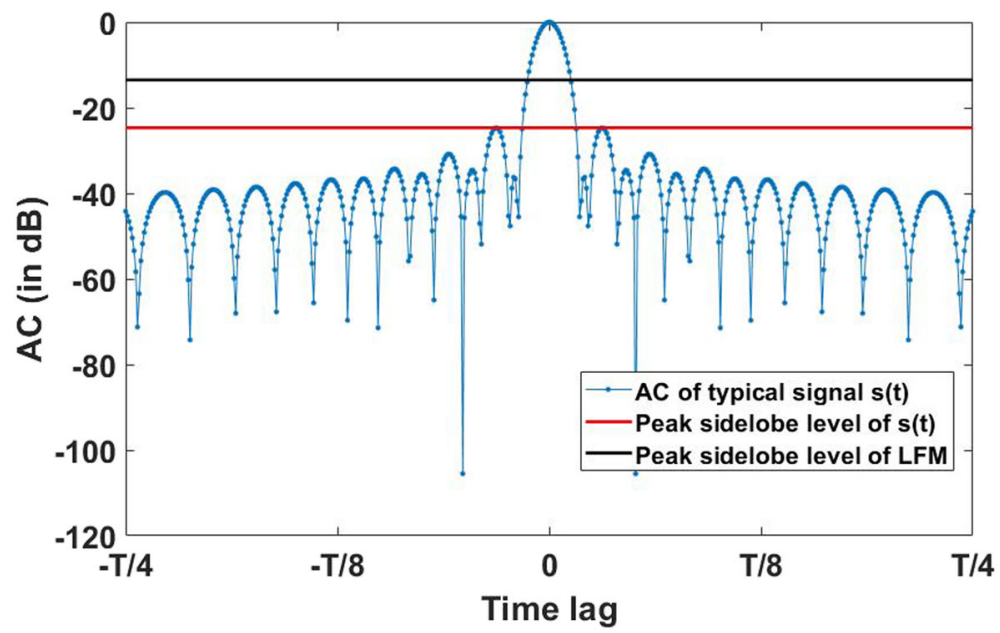

Fig. 4 AC of the typical frequency function shown in Fig. 1. The red (lower) line shows the peak sidelobe level of the typical NLFM signal. The black (upper) line is the peak sidelobe level of LFM, which is significantly higher than that of NLFM

include here. Such signals can reasonably viewed as variation [6] of the signals that we do include for comparison or the signal models that require more information to recreate $[8,9]$ for comparison.

\subsection{Odd polynomial frequency signal: $\boldsymbol{\Omega}_{A}$}

Our proposed polynomial frequency function is

$$
f(t)=\zeta B \sum_{k=0}^{N} p_{k}(2 t / T)^{N-k}, \frac{-T}{2} \leq t \leq \frac{T}{2} .
$$

Since the frequency function in the signal set $\Omega$ is antisymmetric, we set the coefficients corresponding to the even powers $p_{N}, p_{N-2}, p_{N-4}, \ldots$ to zero and optimize the coefficients corresponding to the odd powers $p_{N-1}, p_{N-3}, p_{N-5}, \ldots$. Optimizing only the odd coefficients helps the optimization routines to focus only on the important parameters. Though higher degree polynomials can result in flexible frequency functions, we observed from our experiments the polynomial degree higher than 11 did not yield improvements in terms of AC characteristics. Eleventh degree polynomial has six odd coefficients and hence has six dimensional freedom. We refer this signal as $6 \mathrm{D}$-OPFS. In this paper, we use the $\mathrm{AC}$ characteristics of $6 \mathrm{D}$-OPFS to compare against other FM signal models. Though we intend most of the signal energy to be within the allowed bandwidth, we intentionally allow small amount signal energy to leak beyond the allowed band to have larger effective bandwidth. This can enhance the AC characteristics of the signal. The parameter $\zeta$ in (8) helps in extending the frequency function beyond the allowed bandwidth range of $[-B / 2, B / 2]$. The parameter set for the 6D-OPFS is given by $\Theta_{A}=$ $\left\{\zeta, p_{1}, p_{3}, p_{5}, p_{7}, p_{9}, p_{11}\right\}$.

\subsection{Asymmetric time exponentiated FM (ATEFM): $\boldsymbol{\Omega}_{B}$}

The frequency function of ATEFM has a single parameter $\Theta_{B}$ with $v \in(1,2]$ used to control the nonlinearity:

$$
f(t, v)=\left\{\begin{array}{l}
\frac{B}{2}\left(\frac{2(t+T / 2)}{T}\right)^{\nu-1}-\frac{B}{2}, \frac{-T}{2} \leq t<0 \\
\frac{B}{2}-\frac{B}{2}\left(\frac{2(T / 2-t)}{T}\right)^{v-1}, 0 \leq t<\frac{T}{2}
\end{array}\right.
$$

This signal is used as an example in [23] to compute an accurate estimate of NLFM signals transmitted by noncooperating radars at low SNRs.

\section{Signal optimization}

For the simulation study, we consider discrete time radar signals of the form $s(t)=A \exp (j \phi(t))$, where the phase $\phi(t)$ is the integral of the frequency function $f(t)$ with sample time values at $t=n T / M$ for $n=0,1, \ldots, M-$ 1 . The signal's AC characteristics depend on the timebandwidth product $B T$ and not on the individual values of pulse duration $T$ and bandwidth $B$. From our simulation experiments, we observed that for different values of $B T$ the relative performance of the AC characteristics of the considered signal models remain the same. Here, we present the simulation results for $B T=100$. We set the design parameters as follows.

1. Available signal bandwidth is $B=10 \mathrm{MHz}$ and the pulse duration of the signal is $T=10 \mu \mathrm{s}$.

2. Number of samples $M=1001$ resulting in the sampling frequency of $F_{s}=M / T=100.1 \mathrm{MHz}$ which conveniently satisfies the Nyquist condition with respect to the chosen signal bandwidth $B$.

From each of the parameterized signal sets $\Omega_{A}$, $\Omega_{B}, \ldots \Omega_{H}$ defined in Section 2, we find the best signal element with respect to $Q(s ; \beta)$ : 


$$
s_{\#}^{*}(\beta)=\underset{s \in \Omega_{\# 0}}{\arg \min } Q(s ; \beta)
$$

where $\beta$ is the weight parameter defined in Eq. (6) and $\# \in$ $\{A, B, C, D, E, F, G, H\}$. The signal set denoted by $\Omega_{A 0}$ is the subset of signals from the signal set $\Omega_{A}$ that satisfy the AC mainlobe width and bandwidth requirements defined in Section 1.1. In a similar manner, we define the other parameterized signal subsets $\Omega_{B 0}, \Omega_{C 0}, \ldots, \Omega_{H 0} . s_{A}^{*}(\beta)$ as the best signal from the signal set $\Omega_{A 0}$ with respect to the cost function $Q(s ; \beta)$. Similarly, $s_{B}^{*}(\beta)$ is the best signal from the parameterized signal set $\Omega_{B 0}$ and so on. To find these best signals $s_{A}^{*}(\beta), s_{B}^{*}(\beta), \ldots, s_{H}^{*}(\beta)$ from each of the signal subsets $\Omega_{A 0}, \Omega_{B 0}, \ldots, \Omega_{H 0}$, we need to find the optimum values for the parameters $\Theta_{A}, \Theta_{B}, \ldots, \Theta_{H}$. We used global optimization routines in Matlab [24] based on simulated annealing and pattern search methods to search for these optimum parameters from which we obtain the best signals $s_{A}^{*}(\beta), s_{B}^{*}(\beta), \ldots, s_{H}^{*}(\beta)$.

For reference, Table 1 shows the signal parameters obtained from the optimization routines for all the signal models. We have also listed the PSLR and ISLR obtained for all the signals using these optimum parameters in Table 2 and Table 3. Figure 5 shows the summary of the cost values $Q\left(s_{A}^{*}, \beta\right), Q\left(s_{B}^{*}, \beta\right), \ldots, Q\left(s_{H}^{*}, \beta\right)$ plotted versus the cost function weight $\beta$. We vary the weight $\beta$ from 0.00 to 1.00 in steps of 0.25 . Note that $\beta=0$ corresponds to minimizing only the ISLR and $\beta=1$ corresponds to minimizing only PSLR. We can observe that the 6DOPFS signal outperforms the other signal models for all the considered weights except when $\beta=1$. For $\beta=1$, Price's 6D-OPFS, Collins and Atkins, Wenzhen and Yan, and Cook's signal designs have cost function close to zero (which can be observed in the last column of Table 2). In this case, Price's signal models achieves the minimum cost.

\section{Discussion on sub-optimality of $s_{A}^{*}(\beta), \ldots, s_{H}^{*}(\beta)$ and simulation results}

In the preceding section, we found the best signals from within the parameterized signal sets $\Omega_{A 0}, \ldots, \Omega_{H 0}$. Note that these signal sets $\Omega_{A 0}, \ldots, \Omega_{H 0}$ are subsets of $\Omega_{0}$. We noticed the signal set $6 \mathrm{D}$-OPFS $\left(\Omega_{A 0}\right)$ and the signal set proposed by Price $\left(\Omega_{D 0}\right)$ outperform the other parameterized signal sets for the cost functions we considered. However, the best overall signal from the signal set $\Omega_{0}$ may not lie in any of $\Omega_{A 0}, \ldots, \Omega_{H 0}$. In order to characterize how good $s_{A}^{*}(\beta), \ldots, s_{H}^{*}(\beta)$ and particularly the optimal signals $s_{A}^{*}(\beta)$ and $s_{D}^{*}(\beta)$ are, we are interested to

Table 1 Table listing signal parameters obtained from optimization routines for $B=1000, T=0.1$, and $M=1001$

\begin{tabular}{|c|c|c|c|c|c|c|}
\hline Signals & $\Theta$ & $\beta=0.00$ & $\beta=0.25$ & $\beta=0.50$ & $\beta=0.75$ & $\beta=1.00$ \\
\hline \multirow[t]{7}{*}{ 6D-OPFS } & $\zeta$ & 1.2 & 1.4 & 0.9969788 & 1.4 & 1.0888194 \\
\hline & $p_{1}$ & 2.9833301 & 3.0322699 & 3.2706690 & 2.3061778 & 3.8595594 \\
\hline & $p_{3}$ & -6.0137280 & -5.7741939 & -6.0897503 & -4.5383562 & -4.9449954 \\
\hline & $p_{5}$ & 4.4237943 & 4.1432707 & 3.8108525 & 3.1916240 & 1.7412935 \\
\hline & $p_{7}$ & -1.3836515 & -1.4752306 & -0.7409933 & -0.8812900 & 0.0453272 \\
\hline & $p_{9}$ & 0.1308622 & 0.3757097 & 0.0982280 & 0.1745349 & 0.3363182 \\
\hline & $p_{11}$ & 0.4617059 & 0.2827204 & 0.4160720 & 0.2849106 & 0.0630924 \\
\hline ATEFM & $v$ & 2.0957537 & 1.7356345 & 1.6155135 & 1.6155135 & 1.4533719 \\
\hline Cook & $n$ & 1 & 4 & 4 & 4 & 4 \\
\hline \multirow[t]{2}{*}{ Price } & $B_{1}$ & 0.8436638 & 0.6848811 & 0.5611050 & 0.4381695 & 0.1296791 \\
\hline & $B_{C}$ & 0.1154728 & 0.1847406 & 0.2379900 & 0.2909956 & 0.3511787 \\
\hline \multirow[t]{2}{*}{ Collins and Atkin } & $\alpha$ & $2.79 \times 10^{-7}$ & 7.9176303 & 0.8057049 & 0.6119288 & 0.8456120 \\
\hline & $\gamma$ & $5.22 \times 10^{-5}$ & 0.3160832 & 1.0382372 & 1.2681669 & 1.4259004 \\
\hline \multirow[t]{2}{*}{ Wenzhen and Yan } & $k_{1}$ & 0.3893824 & 0.3987516 & 0.3896719 & 0.2541758 & 0.1094573 \\
\hline & $k_{2}$ & 2.0355661 & 2.0105194 & 2.0347858 & 2.4453675 & 3.0024526 \\
\hline \multirow[t]{2}{*}{ Vizitiu-Arcsin } & $\Delta f$ & 0.6324528 & 0.4586289 & 0.4500549 & 0.4297516 & 0.4243944 \\
\hline & $\Delta t$ & 5.9713625 & 6.3463664 & 6.3915436 & 6.5225125 & 6.5638456 \\
\hline \multirow[t]{3}{*}{ Vizitiu-Power } & $\Delta f$ & 0.5602052 & 0.3627173 & 0.2660381 & 0.2117072 & 0.2412588 \\
\hline & $\Delta t$ & 6.0205251 & 6.1973325 & 5.6443412 & 5.0191368 & 5.4134402 \\
\hline & $m$ & 0.6472525 & 0.8361837 & 1.6453131 & 4.1791038 & 2.2394050 \\
\hline
\end{tabular}


Table 2 PSLR of the parameterized signals computed with the parameters obtained from optimization routines

\begin{tabular}{|c|c|c|c|c|c|}
\hline Signals & $\beta=0.00$ & $\beta=0.25$ & $\beta=0.50$ & $\beta=0.75$ & $\beta=1.00$ \\
\hline 6D-OPFS & -14.6434 & -25.4724 & -33.7720 & -33.2736 & -51.7987 \\
\hline ATEFM & -12.2009 & -18.8418 & -23.6508 & -23.6508 & -24.7634 \\
\hline Cook & -21.6920 & -34.4314 & -34.4314 & -34.4314 & -34.4314 \\
\hline Price & -19.7837 & -24.3293 & -28.8422 & -34.3562 & -59.5167 \\
\hline Collins and Atkins & -13.4874 & -21.2493 & 24.4017 & -29.1210 & -39.1003 \\
\hline Wenzhen and Yan & -23.9121 & -23.8514 & -23.9103 & -32.5355 & -50.0584 \\
\hline Vizitiu-Arcsin & -16.5711 & -18.1255 & -18.1276 & -18.1307 & -18.1306 \\
\hline Vizitiu-Power & -16.7527 & -18.1670 & -18.2869 & -18.3328 & -18.1368 \\
\hline
\end{tabular}

estimate the fraction of signals in $\Omega_{0}$ that is outperformed by $s_{A}^{*}(\beta)$ and $s_{D}^{*}(\beta)$. To do this, we run an experiment by randomly generating signals in $\Omega$ according to a uniform distribution.

\subsection{Random signal experiment design}

In our experiment, we will draw $L$ random signals and, assuming that none of these outperforms the reference signal $s^{*}$, assess what this tells us about the (true) fraction $p$ of $\Omega_{0}$ that outperforms $s^{*}$. Let $\hat{p}$ be an estimate of $p$. If the probability of $s^{*}$ outperforming the $L$ samples is $1-\eta$ (under the assumption that $\hat{p}$ is the fraction of $\Omega_{0}$ that outperforms $s^{*}$ ), we say that we have confidence $\eta$ that $p<\hat{p}$. Therefore, in order to have confidence at least as large as $\eta$, we desire

$$
(1-\hat{p})^{L} \leq(1-\eta) .
$$

Taking natural logarithm on both sides and dividing by $L$ yields

$$
\ln (1-\hat{p}) \leq \frac{1}{L} \ln (1-\eta)
$$

Since for $p$ small $\ln (1-\hat{p}) \approx-\hat{p}$, we can rearrange this expression to yield the estimate $\hat{p}$ as a function of $L$ and $\eta$ as

$$
\hat{p}(L, \eta)=\frac{-\ln (1-\eta)}{L}
$$

(the smallest value for $\hat{p}$ satisfying the inequality and thus achieving the desired confidence level). One may also express this as the number of trials $L$ needed to attain confidence $\eta$ for a probability size $\hat{p}$ :

$$
L(\hat{p}, \eta)=\frac{\ln (1-\eta)}{-\hat{p}}
$$

(the smallest $L$ satisfying the inequality). With a confidence level of $\eta=95 \%$, the above expressions yield (approximately)

$$
\begin{aligned}
& \hat{p}(L)=3 / L ; \\
& L(\hat{p})=3 / \hat{p} .
\end{aligned}
$$

The first of these equations corresponds to the "rule of three" [25].

\subsection{Generation of random signals from $\Omega_{0}$}

We wish to have a means to generate a random signal $s(t)$ from within $\Omega_{0}$ according to a uniform probability distribution function. The time variable $t$ is quantized so that

$$
t \in\{-T / 2,-T / 2+T / M,-T / 2+2 T / M, \ldots, T / 2\}
$$

\begin{tabular}{|c|c|c|c|c|c|}
\hline Signals & $\beta=0.00$ & $\beta=0.25$ & $\beta=0.50$ & $\beta=0.75$ & $\beta=1.00$ \\
\hline 6D-OPFS & 8.7501 & 8.9490 & 9.0546 & 9.0692 & 13.1738 \\
\hline ATEFM & 9.4972 & 9.9480 & 10.3648 & 10.3648 & 11.2750 \\
\hline Cook & 10.6062 & 10.6093 & 10.6093 & 10.6093 & 10.6093 \\
\hline Price & 8.8081 & 8.9610 & 9.1442 & 9.3659 & 10.8203 \\
\hline Collins and Atkins & 9.5269 & 9.8189 & 10.0512 & 10.3953 & 12.6246 \\
\hline Wenzhen and Yan & 9.0983 & 9.0792 & 9.0977 & 9.6324 & 11.7304 \\
\hline Vizitiu-Arcsin & 8.9193 & 8.9479 & 8.9489 & 8.9504 & 8.9506 \\
\hline Vizitiu-Power & 8.9239 & 8.9612 & 9.0006 & 9.0504 & 9.0186 \\
\hline
\end{tabular}

We will also assume a quantized set of frequency values:

$$
f(t) \in\{-B / 2,-B / 2+B / N,-B / 2+2 B / N, \ldots, B / 2\} .
$$

Table 3 ISLR of the parameterized signals computed with the parameters obtained from optimization routines 


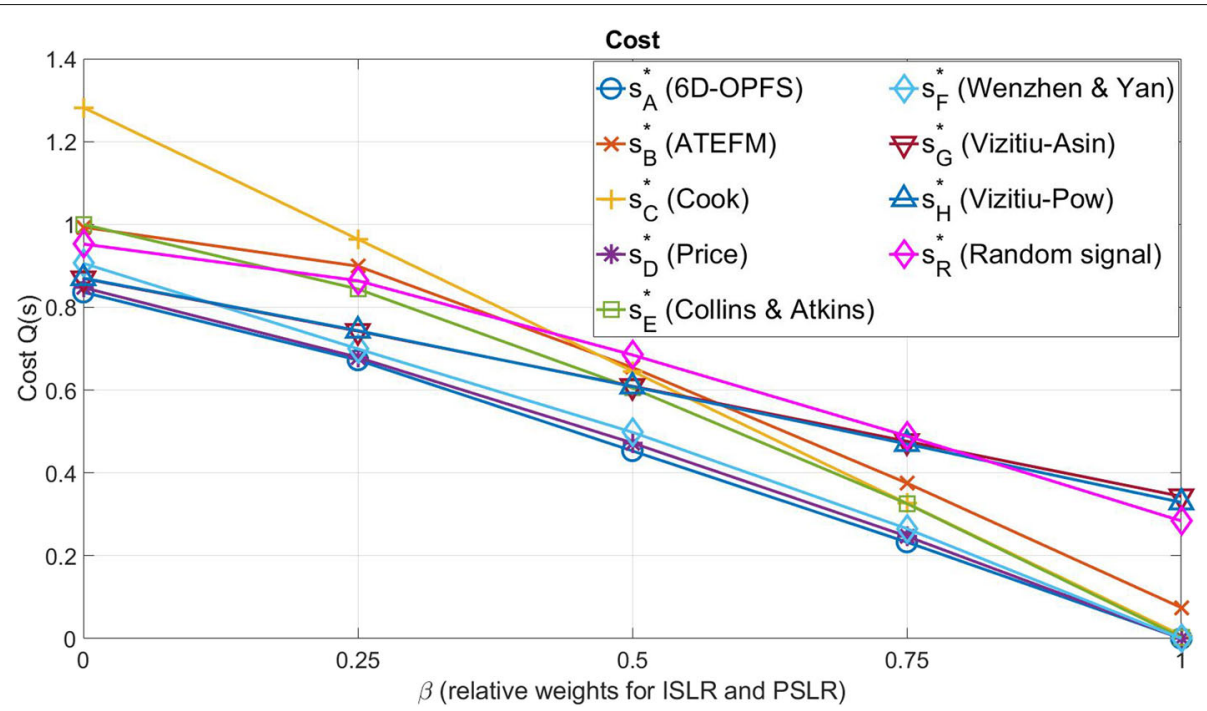

Fig. 5 Performance curves are labeled $s_{A^{\prime}}^{*} s_{B^{\prime}}^{*}$ etc. in reference to the subsets defined in Section 2 . The curve $s_{R}^{*}$ shows the best performance from the randomly generated signals of Section 4

Let $t_{m}=-T / 2+m T / M$. With $f\left(t_{m}\right)=-B / 2+$ $n(m) B / N$, we will for ease of notation refer to the pair $\left(t_{m}, f\left(t_{m}\right)\right)$ as $(m, n(m))$. Given that a frequency $f(t)$ passes through $(m, n(m))$, there is some finite number $K$ of different ways for $f(t)$ to continue to $f(T / 2)=B / 2$ while satisfying the conditions required of $\Omega$. Let us denote $K(m, n)$ as the number of signals that pass through $(m, n)$ in this quantized version of $\Omega_{0}$. Then under a uniform probability distribution, the probability that $f(t)$ passes through $(m+1, n(m+1))$ given that it passes through $(m, n(m))$ is

$\operatorname{Pr}((m+1, n(m+1)) \mid(m, n(m)))=\frac{K(m+1, n(m+1))}{\sum_{k=n(m)}^{N} K(m+1, k)}$.

Note that the expression (13) reflects that $f(t)$ must be nondecreasing. To be able to generate signals in $\Omega_{0}$ randomly, we need to determine $K(m, n)$ for all $m=$ $1, \ldots, M-1$ and all $n$. This is done working from $m=M-$ 1 (corresponding to one step shy of $t=T / 2$ ) backwards to $m=0$ as follows:

1. $K(M-1, n)=1$ for all $n=0,1, \ldots, N$.

2. For $n=N-2$ to

$$
n=1, K(m, n)=\sum_{k=n}^{N} K(m+1, k) \text {. }
$$

The validity of step 1 stems from the fact that all $f(t)$ satisfy $f(T / 2)=B / 2$, so that no matter what the value of $n(M-1)$, there is only one possible choice for $n(M)$. The validity of step 2 is seen by noting that if $f(t)$ passes through $(m, n)$, then the frequency index at time index $m+1$ must lie in $\{n, n+1, \ldots, N\}$, so that the total number of signals passing through $(m, n)$ is the sum of the total number of signals passing through each of $(m+1, n),(m+$ $1, n+1), \ldots,(m+1, N)$. Note also that the total number of quantized $f(t)$ that can be generated this way is

$$
K_{0}=\sum_{n=0}^{N} K(1, n)
$$

We will demonstrate this process of computing the number of frequency paths $K(m, n)$ going through $\left(t_{m}, f\left(t_{m}\right)\right)$ with an example where $M=6$ and $N=5$. The final results of this example are shown in Fig. 6. Due to the antisymmetric nature of the frequency function, it is enough to generate only the first half of the frequency function. Each cell shows the values of $(m, n)$ above the value $K(m, n)$. Hence, for this example, the frequency starts at $-B / 2$ at time $-T / 2$ denoted by location $\left(t_{0}, f_{0}\right)$ and ends at frequency 0 at time 0 denoted by location $\left(t_{5}, f_{4}\right)$. To compute the number of frequency paths through each $\left(t_{m}, f_{n}\right)$ locations, we start from the destination $\left(t_{5}, f_{4}\right)$. As given in step 1 , the number of paths from time $t_{4}$ is $K(4, n)=1$ for $n=0,1,2,3,4$, which we denote in red font in locations $\left(t_{4}, f_{0}\right)$ to $\left(t_{4}, f_{4}\right)$ in the fourth column of the Fig. 6. The number of frequency paths from the time points $t_{3}$ to $t_{0}$ are computed as in step 2. For example, the number of frequency paths through $\left(t_{3}, f_{3}\right)$ is two to one is $\left(t_{3}, f_{3}\right) \rightarrow\left(t_{4}, f_{3}\right) \rightarrow\left(t_{5}, f_{4}\right)$, second is $\left(t_{3}, f_{3}\right) \rightarrow$ $\left(t_{4}, f_{4}\right) \rightarrow\left(t_{5}, f_{4}\right)$. We compute this number from step 2 as $K(3,3)=K(4,3)+K(4,4)=2$. By following this procedure, we can compute the number of possible frequency paths for all $\left(t_{m}, f_{n}\right)$ to $\left(t_{5}, f_{4}\right)$ is the sum of frequency paths from $\left(t_{m+1}, f_{n}\right),\left(t_{m+1}, f_{n+1}\right) \ldots\left(t_{m+1}, f_{4}\right)$. At time $t_{0}$, the only possible frequency location is $f_{0}$, since it is the start of the frequency function. To compute the number 


\begin{tabular}{|c|c|c|c|c|c|c|c|c|}
\hline & & & $t_{0}$ & $t_{1}$ & $t_{2}$ & $t_{3}$ & $t_{4}$ & $t_{5}$ \\
\hline$f_{4}$ & & 0 & & $\begin{array}{c}(1,4) \\
1\end{array}$ & $\begin{array}{c}(2,4) \\
1\end{array}$ & $\begin{array}{c}(3,4) \\
1\end{array}$ & $\begin{array}{c}(4,4) \\
1\end{array}$ & $(5,4)$ \\
\hline$f_{3}$ & & G & & $\begin{array}{c}(1,3) \\
4\end{array}$ & $\begin{array}{c}(2,3) \\
3\end{array}$ & $\begin{array}{c}(3,3) \\
2\end{array}$ & $\begin{array}{c}(4,3) \\
1\end{array}$ & \\
\hline$f_{2}$ & $\begin{array}{l}n \\
11 \\
Z\end{array}$ & 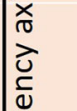 & & $\begin{array}{c}(1,2) \\
10\end{array}$ & $\begin{array}{c}(2,2) \\
6\end{array}$ & $\begin{array}{c}(3,2) \\
3\end{array}$ & $\begin{array}{c}(4,2) \\
1\end{array}$ & \\
\hline$f_{1}$ & & 总 & & $\begin{array}{c}(1,1) \\
20\end{array}$ & $\begin{array}{c}(2,1) \\
10\end{array}$ & $\begin{array}{c}(3,1) \\
4\end{array}$ & $\begin{array}{c}(4,1) \\
1\end{array}$ & \\
\hline $\mathrm{f}_{0}$ & & $-B / 2$ & $\begin{array}{c}(0,0) \\
70\end{array}$ & $\begin{array}{c}(1,0) \\
35\end{array}$ & $\begin{array}{c}(2,0) \\
15\end{array}$ & $\begin{array}{c}(3,0) \\
5\end{array}$ & $\begin{array}{c}(4,0) \\
1\end{array}$ & \\
\hline & & & -T/2 & & \multicolumn{2}{|c|}{ Time axis } & & 0 \\
\hline & & & & & \multicolumn{2}{|c|}{$M=6$} & & \\
\hline
\end{tabular}

Fig. 6 All possible monotonically increasing frequency functions with six discrete time values and five discrete frequency values. Number of possible frequency paths for the given (time, frequency) combination is given by the number written below in red font. All frequency paths start at $(0,0)$ and ends at $(5,4)$

of frequency paths from $\left(t_{0}, f_{0}\right)$ to $\left(t_{4}, f_{5}\right)$, we add all the frequency paths from $\left(t_{1}, f_{1}\right),\left(t_{1}, f_{2}\right),\left(t_{1}, f_{3}\right)$, and $\left(t_{1}, f_{4}\right)$ as

$$
K(0,0)=\sum_{n=0}^{4} K(1, n)=35+20+10+4+1=70,
$$

which is shown in lower left location in Fig. 6.

Once we have created the number of frequency paths table, the next step is to generate a frequency function. The frequency function starts from location $\left(t_{0}, f_{0}\right)$. At this location, there are 70 possible frequency functions. Thity-five of those frequency functions go through location $\left(t_{1}, f_{0}\right), 20$ of them go through $\left(t_{1}, f_{1}\right), 10$ of them go through $\left(t_{1}, f_{2}\right)$, four of them go through $\left(t_{1}, f_{3}\right)$, and one of them goes through $\left(t_{1}, f_{4}\right)$. To randomly pick a frequency path with uniform probability distribution, we choose a random integer $\eta$ from 1 to 70 inclusive with uniform distribution. $\eta$ will determine the choice of frequency location at time $t_{1}$ as given below:

$$
f\left(t_{1}\right)=\left\{\begin{array}{l}
f_{0}, \text { if } 1 \leq \eta \leq 35 \\
f_{1}, \text { if } 36 \leq \eta \leq 55 \\
f_{2}, \text { if } 56 \leq \eta \leq 65 \\
f_{3}, \text { if } 66 \leq \eta \leq 69 \\
f_{4}, \text { if } \eta=70 .
\end{array}\right.
$$

From the selected frequency location at time $t_{1}$, we repeat this procedure for randomly selecting a frequency value at $t_{2}$. For example, say the frequency location at $t_{1}$ is $f_{2}$. From this location, 10 frequency paths are possible as shown in the Fig. 6. Now we choose a random integer $\eta$ from 1 to 10 inclusive and the frequency location at time $t_{2}$ is

$$
f\left(t_{2}\right)=\left\{\begin{array}{l}
f_{2}, \text { if } 1 \leq \eta \leq 6 \\
f_{3}, \text { if } 7 \leq \eta \leq 9 \\
f_{4}, \text { if } \eta=10 .
\end{array}\right.
$$

Note in this case, the frequency values $f_{0}$ and $f_{1}$ are not considered at $t_{2}$ due to the monotonically increasing characteristic of the frequency function. We repeat this procedure by selecting $\eta$ with uniform probability distribution for the remaining time instances till the frequency function reaches the destination $\left(t_{5}, f_{4}\right)$ to get the first half of the frequency function. We obtain the complete frequency function as

$$
f(m)= \begin{cases}f(m), & \text { if } 1 \leq m \leq(M+1) / 2 \\ -f(M+1-m), & \text { if }(M+1) / 2+1 \leq m \leq M\end{cases}
$$

for odd $M$ and

$$
f(m)= \begin{cases}f(m), & \text { if } 1 \leq m \leq M / 2 \\ -f(M+1-m), & \text { if } M / 2+1 \leq m \leq M .\end{cases}
$$

for even $M$.

Using this frequency function, we obtain the associated FM signal as

$$
s(m)=\exp \left(2 \pi j \sum_{l=1}^{m} f(l) \Delta t\right) .
$$

$\Delta t$ is the time interval between the consecutive frequency points. The characteristics of the signal $s$ may not be suitable for radar applications. Hence, we include the signal 
$s$ in the signal set $\Omega_{0}$ only if it satisfies the AC mainlobe width and bandwidth conditions mentioned in (1.1).

\subsection{Experiment parameters and results}

Using the procedure from Subsection 4.2, we generated $L=10^{6}$ random signals from the signal set $\Omega_{0}$ with bandwidth $B=1000$, time width $T=0.1$, and number of samples $M=1001$. We find the best randomly picked signal with respect to the cost metric $Q(s ; \beta)$ for different weights $\beta$. The magenta curve (solid line with diamond shaped markers) in Fig. 5 corresponds to the cost of the best random signals selected for each weight $\beta$. We can observe that the performance of the best random signals are in the same range as most of the parameterized signal sets. But none of these random signals outperform the best low dimensional signal sets for all the considered weights (6D-OPFS signal for $\beta=0,0.25,0.50,0.75$ and Price's signal for $\beta=1.00$ ). Hence, as we argued in the beginning of this section, with $95 \%$ of confidence level, we estimate that 6D-OPFS and Price's signal outperform a fraction of at least $\left(1-\frac{3}{10^{6}}\right)=99.9997 \%$ of the random signals from the signal set $\Omega_{0}$. From the simulation, we can infer that searching for the best radar signal from the 6D-OPFS and Price's signal models require much less computational resources with small degradation in performance.

\section{Conclusion}

In this paper, we searched for the best FM signal with primary objective of minimizing AC sidelobes that are characterized by PSLR and ISLR. Apart from the AC sidelobe levels, the signal has to satisfy other constraints such as AC mainlobe width and IBER. The frequency functions of the FM signals proposed in the literature have monotonically increasing frequency functions with antisymmetry around the temporal midpoint. We referred to the set of signals with such frequency function and also satisfying the AC mainlobe width and IBER constraints as $\Omega_{0}$. Many researchers have proposed parameterized FM signals that are in subsets of $\Omega_{0}$. We searched for the best FM signal from these parameterized signal subsets of $\Omega_{0}$. To find the best signal, we defined cost functions that are convex combinations of PSLR and ISLR with the objective of penalizing the AC sidelobes. We found that the 6D-OPFS signal model proposed in this paper outperforms other signal models for most of the convex combinations and Price's signal model outperforms other signal models for $\beta=1$. But the best radar signal may be from the random signal element of the signal set $\Omega_{0}$ and not from the parameterized signal subsets. It is informative to find the proportion of the random signal elements that 6D-OPFS and Price's signal outperforms with certain confidence interval. From the argument we presented in Section 4, we expect with
95\% confidence that 6D-OPFS and Price's signals outperform at least $99.9997 \%$ of the randomly picked signals from $\Omega_{0}$.

\section{Appendix}

\section{Cook's NLFM Using SPP: $\Omega_{C}$}

Cook [1] designed NLFM signals whose PSDs have a shape of $\cos ^{n}(f)$. In his paper Cook presented the group delays as a function of frequency for $n=1,2,3,4$. With $\tau_{n}(f)$ denoting the group delay for a given value of $n$, we have

$$
\begin{aligned}
\tau_{1}(f)= & \frac{T}{2} \sin \left(\frac{\pi f}{B}\right) \\
\tau_{2}(f)= & T\left[\frac{f}{1.21 B}+\frac{1}{2 \pi} \sin \left(2 \pi \frac{f}{1.21 B}\right)\right], \\
\tau_{3}(f)= & \frac{T}{4} \sin \left(\frac{\pi f}{1.4 B}\right)\left[\cos ^{2}\left(\frac{\pi f}{1.4 B}\right)+2\right], \\
\tau_{4}(f)= & T\left[\frac{f}{1.57 B}+\frac{1}{2 \pi} \sin \left(2 \pi \frac{f}{1.57 B}\right)\right. \\
& \left.+\frac{2}{3 \pi} \cos ^{3}\left(\frac{\pi f}{1.57 B}\right) \sin \left(\frac{\pi f}{1.57 B}\right)\right] .
\end{aligned}
$$

The frequency function $f(t)$ is given by the inverse function of group delay $\tau_{n}(f), \tau_{n}^{-1}(t)$ :

$$
f(t)=\tau_{n}^{-1}(t) .
$$

\section{Price's NLFM: $\boldsymbol{\Omega}_{D}$}

Price's NLFM [2] has a two dimensional parameter $\Theta_{D}=$ $\left[B_{L} B_{C}\right]$ to control the nonlinearity of the frequency function,

$$
f\left(t, B_{L}, B_{C}\right)=\frac{t}{T}\left(B_{L}+\frac{B_{C}}{\sqrt{1-4 t^{2} / T^{2}}}\right), \frac{-T}{2} \leq t \leq \frac{T}{2} .
$$

These parameters also control the signal bandwidth. We modify this frequency expression slightly to

$$
f\left(t, B_{l}, B_{c}\right)=B \frac{t}{T}\left(B_{l}+\frac{B_{c}}{\sqrt{1-4 t^{2} / T^{2}}}\right), \frac{-T}{2} \leq t \leq \frac{T}{2},
$$

where $B_{l}=B_{L} / B$ and $B_{c}=B_{C} / B$. This change makes the parameter search space independent of the signal bandwidth. This change also reduces the parameter space which will facilitate the optimization routines to converge on more appropriate parameter set.

\section{Collins and Atkins NLFM: $\boldsymbol{\Omega}_{E}$}

In [5], Collins and Atkins proposed a NLFM frequency function that has two parameters $\Theta_{E}=\left[\begin{array}{ll}\alpha & \gamma\end{array}\right]$ to con- 
trol the nonlinearity and suggested that parameter values around $[0.5,1.4]$ minimizes the peak sidelobe level.

$f(t, \alpha, \gamma)=\frac{B}{2}\left[\frac{\alpha \tan (2 \gamma t / T)}{\tan \gamma}+\frac{2(1-\alpha) t}{T}\right], \frac{-T}{2} \leq t \leq \frac{T}{2}$.

They also proposed an amplitude weighting function to reduce the sidelobe level. It is possible to find amplitude weighting functions for the other NLFM signal models also to reduce the sidelobes. That activity is beyond the scope of this paper and we will limit the signals to have constant envelope so that we can have fair comparison with the other signal models.

\section{Wenzhen-Yan: $\boldsymbol{\Omega}_{\boldsymbol{F}}$}

Wenzhen and Yan [7] designed a NLFM frequency function with the intention of achieving a PSD whose shape resembles a Blackman window [26]. Such a PSD will result in small AC sidelobes. Their frequency function

$$
f\left(t, k_{1}, k_{2}\right)=B k_{1} \tan \left(k_{2} t / T\right)
$$

has two parameters $\Theta_{F}=\left[\begin{array}{ll}k_{1} & k_{2}\end{array}\right]$. Wenzhen and Yan suggested the parameter values around [0.1171 2.607] to minimize AC sidelobes.

\section{Vizitiu NLFMs: $\boldsymbol{\Omega}_{G}$ and $\boldsymbol{\Omega}_{H}$}

In [13], Vizitiu proposed two NLFM frequency functions that belong to the family of distorted LFM signal models. The first frequency function has two parameters $\Theta_{G}=$ [ $\delta f \delta t]$ in an arcsin based distortion function and is shown in (30) below. The second frequency function has three parameters $\Theta_{H}=\left[\begin{array}{lll}n & \delta f & \delta t\end{array}\right]$ and a $t^{n}$ based distortion function and is shown below in (31).

$$
f(t, \delta f, \delta t)= \begin{cases}\frac{2}{\pi} \delta f \arcsin \left(\frac{t-\delta t}{\delta t}\right)-\frac{B}{2}+\frac{B}{T} \delta t, & t \in(0, \delta t], \\ -\frac{B}{2}+\frac{B}{T} t, & t \in(\delta t, T-\delta t], \\ \frac{B}{2}-\frac{B}{T} \delta t+\frac{2}{\pi} \delta f \arcsin \left(\frac{t-T+\delta t}{\delta t}\right), & t \in(T-\delta t, T] .\end{cases}
$$

$$
f(t, m, \delta f, \delta t)= \begin{cases}\delta f\left(\frac{t}{\delta t}\right)^{m}-\left(\frac{B}{2}+\delta f\right)+\frac{B}{T} \delta t, & t \in(0, \delta t], \\ -\frac{B}{2}+\frac{B}{T} t, & t \in(\delta t, T-\delta t] \\ \frac{B}{2}-\frac{B}{T} \delta t+\delta f-\delta f\left(\frac{T-t}{\delta t}\right)^{m}, & t \in(T-\delta t, T] .\end{cases}
$$

\section{Abbreviations}

AC: Auto correlation; AF: Ambiguity function; ATEFM: Antisymmetric time exponentiated frequency modulation; FM: Frequency modulation; IBER: In-band energy ratio; ISLR: Integrated sidelobe ratio; LFM: Linear frequency modulation; MF: Matched filter; MLW: Main lobe width; NLFM: Nonlinear frequency modulation; OPFS: Odd polynomial frequency signal; PSD: Power spectral density; PSLR: Peak-to-sidelobe ratio; SPP: Stationary phase principle

\section{Acknowledgements}

Not applicable

\section{Authors' contributions}

Both authors contributed equally to the conception of the paper. SA was responsible in creating the simulation results and drafting the paper. GAW was responsible in interpreting the simulation results and substantially revised the draft.

\section{Funding}

Not applicable.

\section{Availability of data and materials \\ Not applicable.}

\section{Competing interests}

The authors declare that they have no conflict of interest.

Received: 22 July 2019 Accepted: 4 December 2019

Published online: 21 December 2019

\section{References}

1. C. E. Cook, A class of nonlinear fm pulse compression signals. Proc. IEEE. 52, 1369-71 (1964). https://doi.org/10.1109/PROC.1964.3393

2. R. Price, in Proc. of URSI National Radio Science Meeting. Chebyshev low pulse compression sidelobes via a nonlinear fm, (Seattle, 1979)

3. N. Levanon, E. Mozeson, Radar Signals. (Wiley-IEEE Press, 2004). http:// books.google.com/books?id=I_2IHI9fVHUC. Accessed 13 Dec 2019

4. H. Meikle, Modern Radar Systems - Second edition. (Artech House Publishers, Norwood, 2008)

5. T. Collins, P. Atkins, Nonlinear frequency modulation chirps for active sonar. IEE Proc. Radar Sonar Navig. 146, 312-316 (1999). https://doi.org/ 10.1049/ip-rsn:19990754

6. C. Leśnik, Nonlinear frequency modulated signal design. Acta Phys. Pol. A 116, 351-354 (2009)

7. Y. Wenzhen, Z. Yan, in Proceedings of 2014 IEEE International Conference on Signal Processing, Communications and Computing (ICSPCC). A novel nonlinear frequency modulation waveform design aimed at side-lobe reduction, (2014), pp. 613-618. https://doi.org/10.1109/ICSPCC.2014. 6986266

8. P. Yichun, P. Shirui, Y. Kefeng, D. Wenfeng, in Proc. IEEE Radar Conf. Optimization design of nlfm signal and its pulse compression simulation, (2005), pp. 383-386. https://doi.org/10.1109/RADAR.2005.1435855

9. J. M. Kurdzo, B. L. Cheong, R. D. Palmer, G. Zhang, in Proceedings of 2014 International Radar Conference. Optimized nlfm pulse compression waveforms for high-sensitivity radar observations, (2014), pp. 1-6. https:// doi.org/10.1109/RADAR.2014.7060249

10. C. E. Cook, J. Paolillo, A pulse compression predistortion function for efficient sidelobe reduction in a high-power radar. Proc. IEEE. 52(4), 377-389 (1964). https://doi.org/10.1109/PROC.1964.2927

11. H. D. Griffiths, L. Vinagre, Design of low-sidelobe pulse compression waveforms. Electron. Lett,. 30(12), 1004-1005 (1994). https://doi.org/10 1049/el:19940644

12. E. D. Witte, H. D. Griffiths, Improved ultra-low range sidelobe pulse compression waveform design. Electron. Lett. 40(22), 1448-1450 (2004). https://doi.org/10.1049/el:20046548

13. I. C. Vizitiu, Some aspects of sidelobe reduction in pulse compression radars using nlfm signal processing. Progress. Electromagn. Res. C. 47 119-129 (2014)

14. L. K. Patton, C. A. Bryant, B. Himed, in Proc. IEEE Radar Conf. Radar-centric design of waveforms with disjoint spectral support, (2012), pp. 269-274. https://doi.org/10.1109/RADAR.2012.6212149

15. G. Lellouch, A. K. Mishra, M. Inggs, Design of ofdm radar pulses using genetic algorithm based techniques. IEEE Trans. Aerosp. Electron. Syst. 52(4), 1953-1966 (2016). https://doi.org/10.1109/TAES.2016.140671

16. S. W. Frost, B. Rigling, in Proceedings of 2012 IEEE Radar Conference. Sidelobe predictions for spectrally-disjoint radar waveforms, (2012), pp. 0247-0252. https://doi.org/10.1109/RADAR.2012.6212145

17. L. K. Patton, C. A. Bryant, B. Himed, in 2012 IEEE Radar Conference. Radar-centric design of waveforms with disjoint spectral support, (2012), pp. 0269-0274. https://doi.org/10.1109/RADAR.2012.6212149

18. H. He, P. Stoica, J. Li, in 2010 2nd International Workshop on Cognitive Information Processing. Waveform design with stopband and correlation 
constraints for cognitive radar, (2010), pp. 344-349. https://doi.org/10. 1109/CIP.2010.5604089

19. S. Alphonse, G. A. Williamson, in 201422 nd European Signal Processing Conference (EUSIPCO). Novel radar signal models using nonlinear frequency modulation (Lisbon, 2014), pp. 1024-1028. http://ieeexplore. ieee.org/stamp/stamp.jsp?tp=\&arnumber=6952344\&isnumber $=6951911$

20. A. Aubry, V. Carotenuto, A. D. Maio, A. Farina, L. Pallotta, Optimization theory-based radar waveform design for spectrally dense environments. IEEE Aerosp. Electron. Syst. Mag. 31(12), 14-25 (2016). https://doi.org/10 1109/MAES.2016.150216

21. H. D. Griffiths, L. Cohen, S. Watts, E. Mokole, C. J. Baker, M. Wicks, S. D. Blunt, Radar spectrum engineering and management: Technical and regulatory issues. Proc. IEEE. 103, 85-102 (2015)

22. S. Boukeffa, Y. Jiang, T. Jiang, in Proceedings of the IEEE 7th International Colloquium on Signal Processing and Its Applications. Sidelobe reduction with nonlinear frequency modulated waveforms, (2011), pp. 399-403. https://doi.org/10.1109/CSPA.2011.5759910

23. S. Alphonse, G. A. Williamson, in Proceedings of IEEE Radar Conference (RadarCon). Estimation of radar signals using passive sensor network, (2015), pp. 1525-1530. https://doi.org/10.1109/RADAR.2015.7131238

24. MATLAB - Version 2017a, Global Optimization Toolbox - Version 3.4.2 (MathWorks, Natick, 2017)

25. F. Tuyl, R. Gerlach, K. Mengersen, The rule of three, its variants and extensions. Int. Stat. Rev. 77(2), 266-275 (2009). https://doi.org/10.1111/j. 1751-5823.2009.00078.x

26. F. J. Harris, On the use of windows for harmonic analysis with the discrete fourier transform. Proc. IEEE. 66(1), 51-83 (1978). https://doi.org/10.1109/ PROC.1978.10837

\section{Publisher's Note}

Springer Nature remains neutral with regard to jurisdictional claims in published maps and institutional affiliations.

\section{Submit your manuscript to a SpringerOpen ${ }^{\circ}$ journal and benefit from:}

- Convenient online submission

Rigorous peer review

- Open access: articles freely available online

- High visibility within the field

- Retaining the copyright to your article

Submit your next manuscript at $>$ springeropen.com 